\begin{tabular}{|c|c|c|c|c|c|}
\hline MUNIBE Antropologia-Arkeologia & $n^{\circ} 69$ & $145-155$ & DONOSTIA & 2018 & ISSN 1132-2217 • eISSN 2172-4555 \\
\hline
\end{tabular}

\title{
Nuevos datos cronológicos sobre la cueva sepulcral neolítica de l'Avellaner (Les Planes d'Hostoles, Girona)
}

\author{
New chronological data about the neolithic burial cave of l'Avellaner \\ (Les Planes d'Hostoles, Girona)
}

PALABRAS CLAVES: Prácticas funerarias, Cronología, Neolítico, Noreste Península Ibérica.

GAKO-HITZAK: Ehortze erritualak, Kronologia, Neolitiko Garaia, Iberiar Penintsulako Ipar-Ekialdea.

KEY WORDS: Funerary Practices, Chronology, Neolithic, Northeast of the Iberian Peninsula

\section{Juan F. GIBAJA(1), Berta MORELL(2), Diego LÓPEZ-ONAINDÍA(3), Aurélie ZEMOUR ${ }^{(4)}$, Àngel BOSCH(5), Josep TARRÚS(6) y Maria EULÀLIA SUBIRÀ(7)}

\section{RESUMEN}

La Cova de l'Avellaner es uno de los contextos arqueológicos del noreste peninsular más conocidos perteneciente cronológicamente a los inicios del neolítico. La presencia de tres cavidades con numerosos restos humanos y su cronología neolítica tan antigua, ha hecho de este yacimiento un lugar de referencia sobre las prácticas funerarias realizadas por aquellas primigenias comunidades agricultoras y pastoras. Las primeras dataciones que se realizaron a inicios de los 90 ofrecieron resultados con márgenes de incertidumbre demasiado altos, que generaban ciertas dudas sobre su validez. Por ello, recientemente decidimos iniciar un programa de dataciones para concretar la cronología de los tres espacios funerarios documentados. Se fecharon tres individuos, uno de cada espacio. Los resultados de esas dataciones y su contextualización en el marco de las primeras prácticas funerarias del noreste peninsular constituyen el objetivo del presente artículo.

\section{LABURPENA}

Neolitiko garaiko hasieran kokatu daitezkeen kontextu arkeologikoetatik hoberen ezagutzen denetako bat dugu Cova de l'Avellaner. Izan ere, haitzulo honek dituen hiru geletan topatzen diren giza aztarna ugari eta aintzineko kronologiak, lehen nekazal eta artzainei buruzko ikerketarako erreferentzi bilakatu dute. Dena den, lortu ziren lehen datazioek zeukaten ziurgarritasun marjin altuek beraien baliozkotazunari buruzko kezkak sortu zituzten. Hori dela eta, datazio programa berri bat hastea erabaki genuen, haitzuloaren hiru eremuen kronologia zehaztu ahal izateko. Helburu honekin, gela bakoitzeko gizabanako baten laginak datatu ziren. Artikulu honen helburuak datazio hauen emaitzak eta hauekin, Iberiar Penintsulako lehen ehortze praktiken testuinguru barruan, egindako interpretazioak azaltzea dira.

\begin{abstract}
Until a few years ago, the available information regarding the funerary practices of the first Neolithic communities was negligible. Currently, this has changed significantly, thanks, particularly, to the works carried out in sites such as Cova Bonica or Can Sadurní. In this context, the Cova de l'Avellaner, the addressed site of this paper, was one of the best-known Early-Neolithic archaeological contexts from the Northeast of the Iberian Peninsula. The site has three cavities with numerous human remains. This exceptional archaeological record and its old chronology have made this cave a reference site of the funerary practices of the first farming societies.

The information provided, both by contexts such as Cova de l'Avellaner, and those currently being excavated, seem to show that the caves were selected during this time as burial sites. Different individuals were buried in those cavities, probably accompanied by different tools, containers and ornaments as grave goods. The problem is that it is practically impossible to assign such elements to each of the dead and even to recognize others who could be part of the sediment that covered the bodies. In any case, in this paper a description of the discovered materials are presented, since their characteristics can help to recognize which type of artefacts could accompany the buried and because they also have an added value as a chronological marker; this is what has helped us to define better the moment in which the burials were practiced. The first radiocarbon dates from Cova de l'Avellaner were made during the nineties and provided too wide margins, so their validity is doubtful For this reason we have recently started a radiocarbon dating programme, in order to determine the chronology of the three funerary spaces
\end{abstract}

\footnotetext{
(1) Intitució Milá i Fontanals (IMF-CSIC). Grupo de Arqueología de las Dinámicas Sociales. C/Egipcíaques 15, 08001 Barcelona, jfgibaja@imf.csic.es (2) Departament de Prehistòria. Universitat Autònoma de Barcelona, 08193 Bellaterra (Cerdanyola del Vallès), Barcelona, morell.berta@gmail.com (3) Grup de Recerca Aplicada al Patrimoni Cultural (GRAPAC). Unitat d'Antropologia Biològica. Facultat de Biociències (Edifici C), Universitat Autònoma de Barcelona. 08193 Bellaterra, Barcelona, diego20lopez@gmail.com

(4) Ausonius-UMR 5607 CNRS, LabEx Sciences Archéologiques de Bordeaux (ANR-10-LABX-52). Maison de l'Archéologie, 8, Esplanade des Antilles, 33607 Pessac Cedex, Francia, aurelie.zemour@u-bordeaux-montaigne.fr

(5) Prehistoriador.Girona. aboschlloret@gmail.com

(6) Prehistoriador. Banyoles. joseptarrus@gmail.com

(7) Grup de Recerca Aplicada al Patrimoni Cultural (GRAPAC). Unitat d'Antropologia Biològica. Facultat de Biociències (Edifici C), Universitat Autònoma de Barcelona. 08193 Bellaterra, Barcelona, Eulalia.Subira@uab.cat
} 
of the cave. Three individuals have been dated, one from each cavity. The main goal of this work is to contextualise the obtained results in the frame of the first funerary practices from the Northeast of the Iberian Peninsula.

The use of the caves as a funerary place disappears at the end of the fifth millennium, when the communities begin to inhumate their individuals recurrently in pits or stone boxes, especially individually. That period is known as "the Pit Burials Culture". Curiously, at the end of the Neolithic and the beginnings of the Chalcolithic, in the last centuries of the IV millennium, caves are once again used as collective burial sites, at the same time dolmens or artificial hypogea also appear as funerary structures.

We are currently working on the materials and human remains that were exhumed in this cave at the end of the 20th century. The magnificent state of conservation of the biotic and abiotic remains has led us to address new analysis that we hope to present in the coming years. Among them, the review of all the graphic and anthropological material, in order to evaluate if there were more buried individuals, but especially how the burial process was, should be highlighted. In this aspect, the so-called Anthropologie de Terrain has much to say. Likewise, we have started a morphology analysis of the dentition of the individuals that should provide information regarding the connections between these communities and other groups.

\section{LOS INICIOS DEL NEOLÍTICO EN EL NORES- TE DE LA PENÍNSULA IBÉRICA: PRÁCTICAS FUNERARIAS Y CRONOLOGÍA}

Este trabajo tiene por objetivo presentar las nuevas dataciones realizadas en una de las cuevas sepulcrales de referencia para el neolítico del noreste de la península ibérica: la Cova de l'Avellaner (Cogolls, les Planes d'Hostoles, la Garrotxa). Excavada entre el 1985 y el 1986, es uno de los pocos contextos en cueva donde se han documentado numerosas inhumaciones, en concreto hasta 19. Por consiguiente, este artículo aporta información suplementaria a la magnífica monografía que se publicó en 1990 en relación al yacimiento y en el que había un apartado sobre la cronología de las inhumaciones documentadas (BOSCH y TARRÚS, 1990).

Las primeras dataciones radiocarbónicas realizadas por método convencional indicaban que alguna de las tres cavidades que conforman la cueva, en concreto la primera (muestras sobre carbones) y la tercera (muestras sobre huesos humanos), tuvieron un uso funerario entre finales del VI y mediados del V milenio cal BC. Ello convertía a la Cova de l'Avellaner en uno de los yacimientos neolíticos más antiguos con restos óseos humanos.

Si bien, desde entonces, diversas inhumaciones en cueva de inicios del neolítico han sido descubiertas en el noreste peninsular, caso de los restos hallados en la Cova de Can Sadurní (Begues, Barcelona) o, más recientemente, en Cova Bonica (Vallirana, Barcelona), siguen siendo muy escasas las evidencias funerarias en este tipo de contextos y durante este periodo (BLASCO et alii, 2005, OMS et alii, 2017). Esta escasez inhumaciones cambiará algunos siglos después, con la proliferación, de manera sistemática, de enterramientos realizados en fosas o en cistas excavadas en el suelo, en lo que se conoce como la Cultura de los Sepulcros de Fosa (GIBAJA, 2004, OMS et alii, 2016).

En este marco, las primeras dataciones realizadas en Cova de l'Avellaner debían ponerse en cuarentena por el tipo de muestra fechada y por el resultado obtenido. Por ello, iniciamos un pequeño programa de dataciones dirigido a fechar uno de los individuos de cada una de las tres cavidades. Pretendíamos cerciorarnos de la cronología de esas tres inhumaciones, tener un primer referente de cuándo podían haberse depositado el resto de cuerpos de cada cavidad sepulcral y situar cronológicamente este tipo de prácticas funerarias en relación, no sólo a las otras cuevas con inhumaciones, sino también al conjunto de enterramientos documentados desde inicios del neolítico hasta el calcolítico.

\section{LA COVA DE L'AVELLANER}

Localizado en el municipio de Les Planes d'Hostoles (Girona), se trata de un yacimiento localizado en una terraza travertínica, en la orilla izquierda del torrente de Cogolls, a unos 430 m. s.n.m. Las cavidades que conforman esta cueva son tres hendiduras naturales dentro de dicha roca travertínica (Latitud norte: $42^{\circ} 04^{\prime}$ 30”, Longitud este: $02^{\circ} 32^{\prime}$ 30) (Fig. 1).

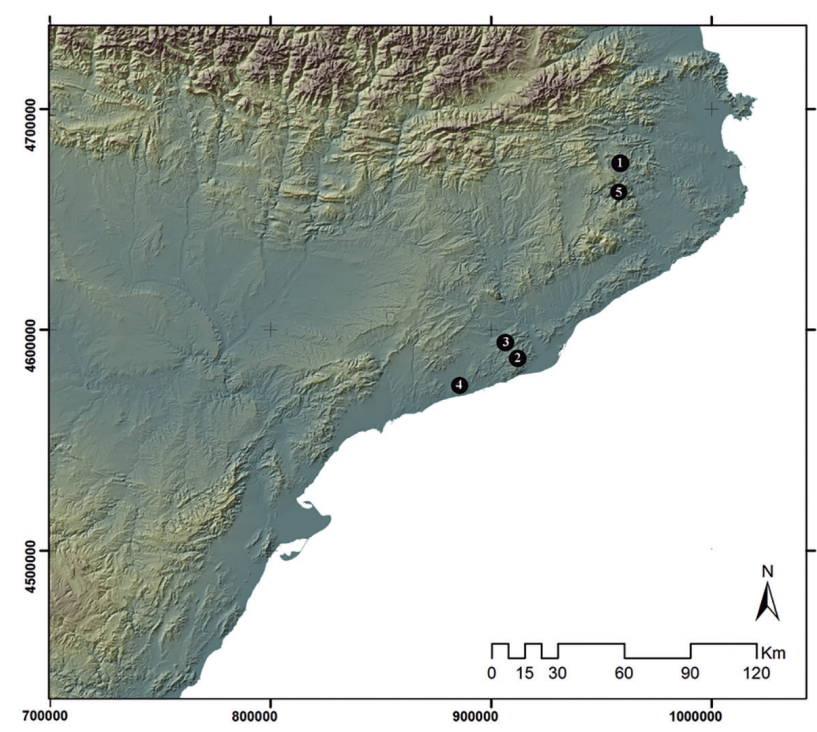

Fig. 1. Situación de yacimientos citados en el texto del noreste peninsular: 1. Cova de l'Avellaner; 2. Cova de Can Sadurní; 3. Cova Bonica; 4. Cova Foradada; 5. Cova del Pasteral / Location of the northeastern Iberian Peninsula sites cited in the text: 1. Cova de l'Avellaner; 2. Cova de Can Sadurní; 3. Cova Bonica; 4. Cova Foradada; 5. Cova del Pasteral.

El 13 de agosto de 1985 se descubre el yacimiento en el curso de una prospección de esa zona a cargo de Josep Tarrús y Àngel Bosch. En noviembre de ese mismo año se inicia la primera campaña centrada en la excavación de la zona-l (con la cavidad sepulcral-1), momento en el cual se comprueba que existe una se- 
gunda zona arqueológica con dos cavidades sepulcrales más. Éstas serán excavadas en 1986 a lo largo de distintas campañas (BOSCH y TARRÚS, 1987, 1990, BOSCH et alii, 1989).

La zona-I estaba formada por dos grietas, una de este a oeste $(1,20 \times 0,40 \mathrm{~m}$. y 1,80 m. de altura) y la otra de norte a sur (1,80 x 0,40 m. y 1,60 m. de altura), dentro de las cuales estaba la cavidad sepulcral-1. Esa cavidad contenía los restos de 8 individuos (4 infantiles, 1 juvenil y 3 adultos) muy revueltos (Fig. 2).

La zona-II contenía dos cavidades sepulcrales parcialmente conservadas: una abierta hacia el sur (cavidad sepulcral-2, de 0,80 x 0,50 m. y 0,95 m. de altura) y la otra al este (cavidad sepulcral-3, de 1,20 x 0,80 m. y 1,80 m. de altura). Ambas estaban separadas por muros de piedra seca. La cavidad sepulcral-2 contenía 6 individuos ( 1 infantil, 4 adultos y 1 senil), mientras que

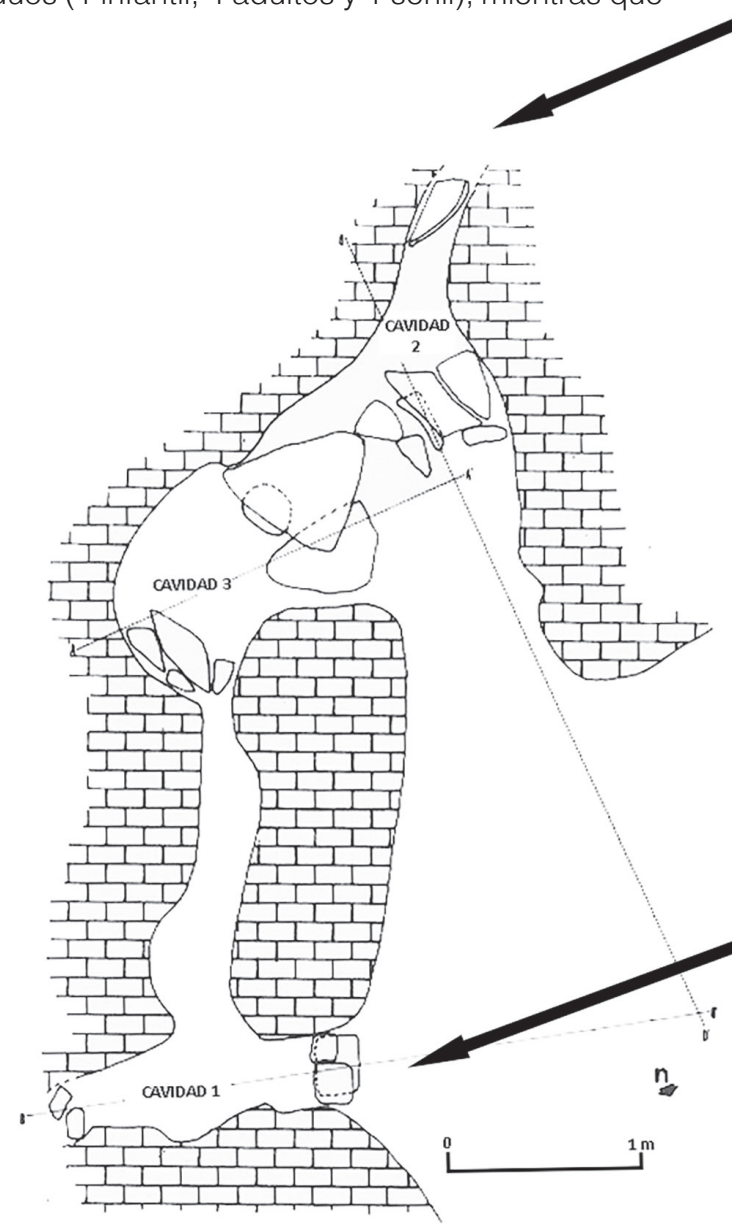

Fig. 2. Planta de la Cova de l'Avellaner: Fotos de la zona-I y zona-II / Floor plan of the Cova de l'Avellaner: Photos of the zone-I and zone-II. en la cavidad sepulcral-3 se hallaron 5 individuos ( 1 infantil, 1 juvenil, 2 adultos y 1 senil). Por encima de la cavidad sepulcral-3, aprovechando una chimenea de la hendidura travertínica, se localizaron los restos de una posible cavidad sepulcral-4, en la que no se documentó ningún resto humano.
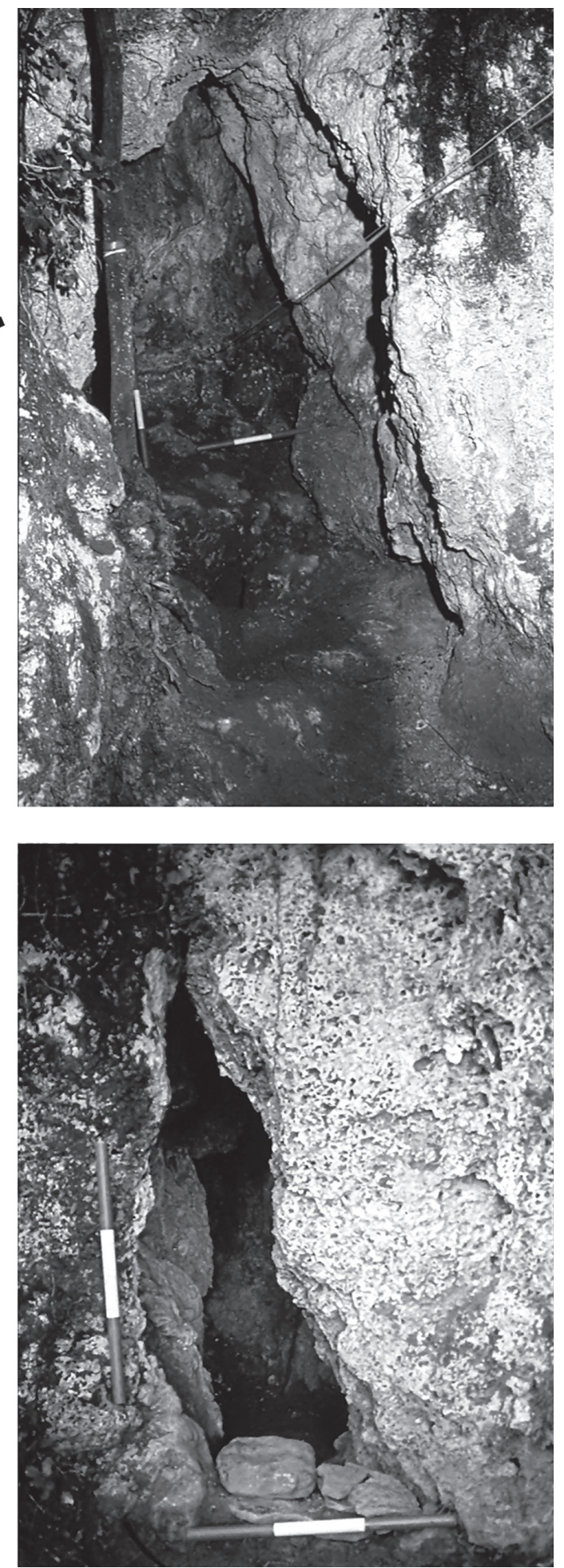
El análisis antropológico realizado por Oriol Mercadal apuntaba que se depositaron como mínimo 19 individuos en esas tres cavidades sepulcrales. A pesar de las remociones provocadas por su uso como madriguera de pequeños mamíferos, se conservó una representación de casi todos los huesos del esqueleto, aunque ninguno apareció completo. A este respecto, habría que tener en cuenta las remociones que entonces se realizaron para ir depositando los cuerpos. En relación al sexo de los individuos, se distinguieron con seguridad 5 masculinos (4 adultos y 1 senil) y 3 femeninos (2 adultos y 1 senil). El resto eran alofisos, es decir, indeterminables (BOSCH et alii, 1989).

En general, el estado de salud de la población inhumada era bueno. Los signos de patología son escasos y corresponden esencialmente a caries, periodontitis y algo de artrosis. El estudio isotópico realizado demuestra que los sujetos consumieron, en especial, recursos vegetales con un complemento de proteínas animales. Estos datos coinciden con los obtenidos sobre otros individuos neolíticos del noreste de la península ibérica (FONTANALS et alii, 2016).

Un análisis genético realizado sobre 7 de los 19 individuos y publicado en 2011 (LACAN et alii., 2011), indicaba que 6 de los individuos analizados eran hombres. Este estudio señalaba una gran variabilidad de haplotipos de ADN mitocondrial (4 haplotipos en esos 7 individuos). Si bien en la península ibérica algunos de esos haplotipos ya estaban presentes en el mesolítico, otros solo están relacionados con los grupos de los primeros agricultores (HERVELLA et alii., 2012; LACAN et alii., 2011; de la Rúa et alii., 2015; SZÉCSÉNYI-NAGY et alii., 2017). En cuanto al estudio del cromosoma $Y$, los datos mostraron que 5 de los 6 individuos masculinos presentaban el haplogrupo G2a. Dicho haplogrupo se ha observado también en individuos de yacimientos neolíticos de Alemania y Francia, lo que, según los autores del estudio, podría estar relacionado con el significativo papel de los individuos masculinos en la expansión del neolítico en el Mediterráneo Occidental europeo (LACAN et alii., 2011).

El rito funerario que se puede deducir en el caso de las pequeñas cavidades sepulcrales de la Cova de l'Avellaner sería el de depósitos, más que inhumaciones, primarias y sucesivas. Es decir, por falta de espacio y de sedimento, suponemos que no fueron enterrados en un hoyo cubierto de tierra, sino simplemente dejados en el suelo rocoso de las cavidades. Ese tipo de rito parece idéntico al documentado, por ejemplo, en el yacimiento francés de la Baume Bourbon (Cabrières, Gard, Languedoc Oriental) (ZEMOUR, 2013).

Las tres cavidades principales funcionarían como contenidos arquitectónicos únicos destinados a la protección de los cuerpos. Probablemente quedarían selladas mediante materiales perecederos o bloques de piedras. Recordemos, que la cavidad sepulcral-1, de la zona-I, presenta un muro de piedra seca que obtura su pequeña entrada y que las cavidades sepulcrales-2 y 3 , dentro de la zona-II, estaban separadas también por diversos bloques que conformaban los dos espacios funerarios. De este modo, podemos afirmar que cada una de las cavidades sepulcrales estaban pensadas para proteger a los cuerpos depositados en ellas, cosa que se consiguió seguramente durante bastante tiempo, hasta que los fenómenos naturales (agua) y la actividad de los pequeños mamíferos removieron los enterramientos hasta que fueron hallados en 1985-1986 (BOSCH y TARRÚS, 1990).

Cada nuevo depósito supuso la remoción, y seguramente la reducción, de los individuos anteriores. En este sentido, la existencia de numerosos huesos humanos y de fauna con cremaciones parciales a baja temperatura $\left(300^{\circ}-350^{\circ}\right)$ nos han inducido a pensar que el fuego era utilizado para conseguir nuevos espacios en la cavidad sepulcral. Actualmente, se ha puesto en marcha una nueva revisión de los restos osteológicos que permitirá aportar nuevos datos sobre la función funeraria del yacimiento. Tal información se incluirá en un estudio comparativo con otros contextos sepulcrales de la tradición Impreso-Cardial del Mediterráneo noroccidental (ZEMOUR, 2014, ZEMOUR et alii, 2017).

Cabe señalar que entre los restos de fauna doméstica (cerdo, oveja-cabra) o salvaje (corzo, ciervo, jabalí) predominaban las extremidades, cosa propia de las ofrendas funerarias por ser las partes con mayor y mejor proporción de carne.

Los objetos manufacturados hallados en las tres cavidades sepulcrales fueron vasos cerámicos, piezas líticas talladas, manos de molinos de piedra, útiles sobre hueso o asta y objetos de adorno elaborados en concha, piedra, hueso o dientes de animales.

Con respecto a la cerámica, se documentaron fragmentos de hasta 41 vasos de tradición epicardial (Fig. 3). La industria ósea fue especialmente rica al hallarse: 2 espátulas-colgantes (procedentes de un omóplato de un gran herbívoro, seguramente un bóvido), 2 punzones y 10 fragmentos de punzón sobre asta de corzo; 1 fragmento de punzón confeccionado sobre metápodo de ovicáprido; 1 objeto fusiforme con el tallo roto (un posible punzón o punta del que desconocemos la especie y el hueso con el que se elaboró); 2 fragmentos de útiles indeterminados con extracciones (de especie no determinable); y 1 arranque de asta de ciervo con extracciones (posiblemente una matriz).

La industria lítica era bastante pobre. La formaban 202 elementos, elaborados en sílex, cristal de cuarzo, cuarzo, lidita y corneana, entre los que únicamente había 12 piezas retocadas (laminillas con retoques marginales, raspadores, muescas, buriles y guijarros o cantos tallados). Completaban el registro lítico 8 núcleos poliédricos y prismáticos y 182 restos de talla.

Los 25 objetos de adorno recuperados en las tres cavidades sepulcrales eran mucho más variados. Así, 

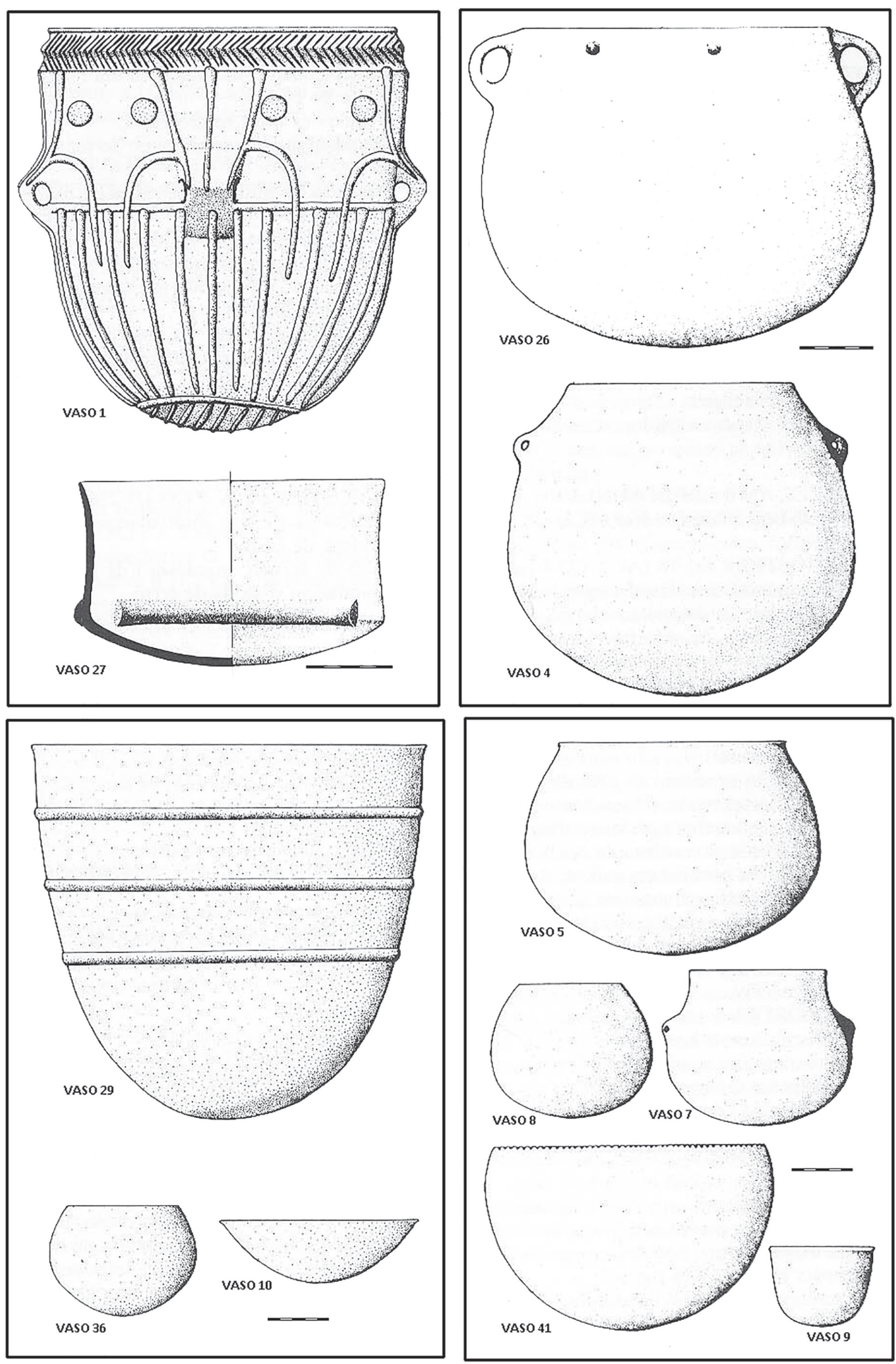

Fig. 3. Principales formas cerámicas (Epicardial reciente) de la Cova de l'Avellaner / Main pottery types (late Epicardial) of the Cova de l'Avellaner. 
se documentaron: 1 brazalete de pectúnculo articulado con doble perforación; 3 discos biperforados en su zona central de pectúnculo; 2 grandes colgantes sobre colmillo de jabalí, uno con una única perforación en su extremo basal y otro igual pero con dos perforaciones (Fig. 4); 1 colgante de roca verde en forma de lágrima (no analizada y que quizás podría tratarse de variscita); 1 colgante pequeño, lenticular y con una perforación trabajado sobre colmillo de jabalí; 1 colgante sobre concha de cardium perforada; 10 cuentas de collar discoidales u ovaladas, de las cuales 7 estaban elaboradas sobre concha, 2 sobre esteatita y 1 sobre calcita; y , finalmente, 6 fragmentos de concha (4 de cardium y 2 de pectúnculo) en proceso de fabricación para obtener cuentas de collar discoidales.

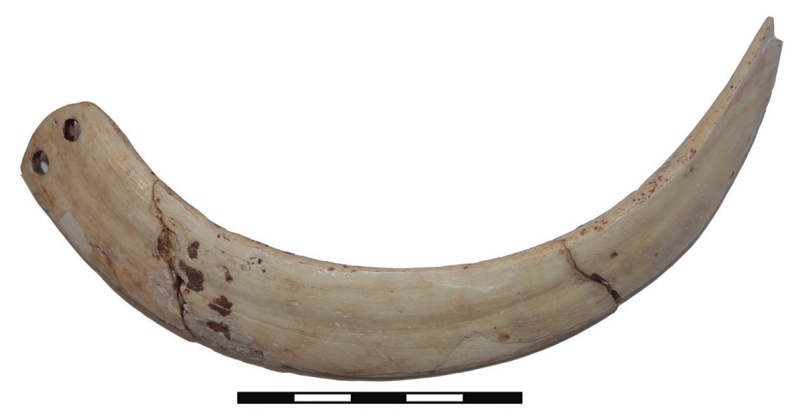

Fig. 4. Colmillo de jabalí biperforado de la cavidad sepulcral-3 (zona-II) / Perforated boar tusk from the sepulchral cavity-3 (zone-II).

Entre la fauna registrada había restos de distintas especies domésticas (oveja-cabra y cerdo) y salvajes (corzo, ciervo y jabalí), que hemos considerado que formaban parte del ajuar funerario. La presencia de algunos restos de perro (Canis familiaris) también podrían vincularse con los materiales dejados como ajuar.

Buena parte de estos recipientes, instrumentos y ornamentos llegaron a las cavidades con los difuntos, algunos depositados ex profeso - como los vasos cerámicos, los objetos de adorno y algunos objetos de hueso, caso de las espátulas y colgantes- y otros quizás como parte del utillaje que habitualmente usaba el inhumado (entre ellos, varios de los útiles líticos tallados y los elaborados en hueso).

Cabe insistir que todo el ajuar funerario era muy homogéneo y encajaba perfectamente con un momento de lo que se conoce como Epicardial final, equivalente a la fase preliminar del Neolítico Medio Inicial del grupo de Montboló. Este momento es especialmente bien conocido en la comarca de la Garrotxa (Olot), donde aparece en los hábitats de Plansallosa-II (Vall del Llierca) (BOSCH et alii, 1998), así como en la Dou y la Codella (Vall d'en Bas) (ALCALDE y SAÑA, 2009). Los útiles recuperados en hueso y piedra tallada, además de los objetos de adorno elaborados en concha, hueso, diente o piedra, son propios del neolítico antiguo, ya en una fase reciente, aunque no son determinantes para una cronología afinada.
En cambio, los fragmentos cerámicos decorados y las formas de los vasos de las cuatro cavidades nos sitúan, sin duda, en inicios del V milenio cal BC. En ellos sólo hallamos un fragmento pequeño con impresiones de peine, y otros tres, minúsculos, con un surco inciso, que podríamos considerar herencia de un Epicardial antiguo o Cardial final. El resto de decoraciones en el material cerámico se reduce a cordones lisos. Finalmente, apuntar la presencia de vasos sin decorar, la mayoría de ellos globulares, que dominan el conjunto.

El descubrimiento de una taza con carena baja provista de una barrita trapezoidal sobre ella, y otro fragmento con un asa tubular vertical, nos indican que las formas y prensiones características del grupo de Montboló ya estaban presentes en la Cova de l'Avellaner. Por otro lado, ninguna de estas formas cerámicas lisas encajaría en un Montboló reciente de finales del $\checkmark$ milenio cal BC ni, aún menos, en un Neolítico Medio Pleno de la primera mitad del IV milenio cal BC, como sería el horizonte, bien conocido, de los Sepulcros de Fosa (BOSCH y TARRÚS, 1990).

Recordemos que tanto el grupo de Montboló en el norte, como el de Molinot en el centro-sur de Cataluña, presentan sus primeras fechas de radiocarbono en la primera mitad del V milenio cal BC. Es el caso de los hábitats de Ca n'Isach, en el Alt Empordà (TARRÚS, 2017), de La Dou, en la Garrotxa (ALCALDE et alii, 2013-2014) y de la cueva de Can Sadurní, en el Baix Llobregat ((BLASCO et alii, 2011). Más tarde experimentarán su apogeo en la segunda mitad del V milenio cal BC, con el inicio de las tumbas con foso de acceso y las cistas con grandes túmulos de Tavertet (MOLIST y CLOP, 2010).

Otro hecho a tener en cuenta es la escasa capacidad de las cavidades sepulcrales de la Cova l'Avellaner. Difícilmente podemos imaginar que su uso pudiera prolongarse más allá de 100-200 años antes de quedar totalmente colmatadas.

\section{NUEVAS DATACIONES}

\subsection{Precedentes}

Hasta ahora, las referencias cronológicas que teníamos de estas cavidades funerarias de la Cova de l'Avellaner procedían, especialmente, de las características morfológicas y decorativas de la cerámica, así como de las dos dataciones realizadas sobre muestras de carbón o hueso humano mediante el método de datación convencional.

En ambos casos, los datos nos remitían a la mitad del V milenio cal BC; es decir, lo que en horizontes culturales para el noreste de la península ibérica sería un neolítico antiguo Cardial final o un Epicardial reciente.

Sin embargo, como hemos comentado, en el marco del proyecto de investigación I+D: "Aproximación a las primeras comunidades neolíticas del NE peninsular a través de sus prácticas funerarias" (HAR2011-23149), 
decidimos poner en marcha un pequeño programa de dataciones dirigido a fechar a uno de los muertos depositados en cada una de las tres cavidades funerarias (Tabla 1). Considerábamos que era necesario hacer nuevas dataciones por dos motivos:

1. Partimos del principio que aquellas dataciones con variaciones superiores a \pm 100 años deben ser tomadas con suma precaución (ZILHÃO, 2001, MORALES et alii, 2010).

2. Las dataciones de carbones suelen ofrecer cronologías más antiguas como resultado del conocido como "efecto de madera vieja". Al desconocer la especie y la parte del árbol de donde procedía el carbón analizado, el resultado de la fecha GAK-12933 nos generaba ciertas dudas (DEAN, 1978, SCHIFFER, 1986, ASHMORE, 1999). Estas dudas se acrecentaron cuando revisando la información se observó que esa datación no provenía de un único carbón, sino de varios fragmentos hallados en la cavidad sepulcral-1.

Ante esta situación decidimos fechar mediante AMS (Accelerator Mass Spectrometry) los restos óseos de una de las inhumaciones de cada cavidad.

\subsection{Cronología de la Cova de l’Avellaner: méto- do y resultados}

Para evaluar los resultados cronométricos obtenidos hemos realizado un conjunto de tests estadísticos dirigidos a conocer el periodo en el que fueron inhumados los individuos fechados. Para ello, en primer lugar, hemos analizado la distribución cronológica del total de dataciones disponibles, tanto las nuevas como las antiguas, mediante la suma de sus distribuciones de probabilidad (WENINGER, 2011, WILLIAMS, 2012). Tal y como podemos observar en el gráfico (Fig. 5), las inhumaciones fueron continuas en el tiempo entre el 5250 y el 4490 cal BC $(2 \sigma)^{1}$, aproximadamente.

Sin embargo, el hecho de trabajar con muestras analizadas mediante métodos diferentes (convencional y AMS), en las que, como ya hemos comentado, las más antiguas presentan desviaciones estándar igual o mayor a \pm 100 y en las que además una de ellas procedía de la unión de varios carbones, nos obligó a evaluar su distribución cronológica por separado para no generar una lectura errónea. Tal y como se puede observar en la figura 6, las muestras más antiguas sitúan la distribución cronológica de los enterramientos en un intervalo temporal más amplio (5190-4430 cal BC) y, por lo tanto, más incierto, que las muestras fechadas mediante AMS (5250-4740 cal BC).

Estos resultados, junto a los problemas citados, nos han llevado a descartar las dos dataciones más antiguas y tomar únicamente los resultados asociados a las tres más recientes.

La prueba de Chi-Cuadrado, que permite evaluar el grado de superposición entre las tres fechas, no ha dado suficiente consistencia estadística. Por consiguiente, en base a los datos disponibles, podemos afirmar que los tres enterramientos no fueron contemporáneos en el tiempo.

\begin{tabular}{|c|c|c|c|c|c|c|c|c|c|c|c|c|}
\hline Laboratorio & $\begin{array}{l}\text { Fecha } \\
\text { BP }\end{array}$ & $\begin{array}{l}\text { Desviación } \\
\text { Estándar }\end{array}$ & Muestra & Procedencia & Referencia & $\begin{array}{c}\text { Calibración } \\
2 \sigma\end{array}$ & $\begin{array}{l}\delta 15 \mathrm{~N} \\
(\%)\end{array}$ & $\begin{array}{c}\delta 13 C \\
(\%)\end{array}$ & C:N & $\% \mathrm{C}$ & $\% \mathrm{~N}$ & $\begin{array}{l}\text { Referencia } \\
\text { Bibliográfica }\end{array}$ \\
\hline GAK12933 & 5920 & 180 & $\begin{array}{c}\text { Grupo } \\
\text { carbones }\end{array}$ & Cavidad 1 & Carbones & $5290-4400$ & & & & & & $\begin{array}{c}\text { Bosch y Tarrús, } \\
1990\end{array}$ \\
\hline UBAR109 & 5830 & 100 & $\begin{array}{l}\text { Hueso } \\
\text { Humano }\end{array}$ & Cavidad 3 & $\begin{array}{l}\text { Huesos } \\
\text { humanos }\end{array}$ & $4935-4460$ & & & & & & $\begin{array}{c}\text { Bosch y Tarrús, } \\
1990\end{array}$ \\
\hline CNA3304.1 & 5941 & 34 & $\begin{array}{c}\text { Hueso } \\
\text { humano }\end{array}$ & Cavidad 1 & $\begin{array}{c}\text { AV-CO I-X } \\
25 \\
\text { (Peroné) }\end{array}$ & $4930-4725$ & 8,47 & $-21,14$ & 3,8 & 30,9 & 9,6 & Inédita \\
\hline CNA3305.1 & 6204 & 34 & $\begin{array}{l}\text { Hueso } \\
\text { humano }\end{array}$ & Cavidad 2 & $\begin{array}{l}\text { AV-CO II- } \\
155 \text { (Tibia) }\end{array}$ & 5290-5055 & 8,75 & $-20,52$ & 3,5 & 36,3 & 12,1 & Inédita \\
\hline CNA3306.1 & 6094 & 34 & $\begin{array}{l}\text { Hueso } \\
\text { humano }\end{array}$ & Cavidad 3 & $\begin{array}{l}\text { AV-CO III- } \\
284 \text { (Radio) }\end{array}$ & $5210-4860$ & 8,60 & $-20,77$ & 3,8 & 33,9 & 10,5 & Inédita \\
\hline
\end{tabular}

Tabla 1: Dataciones radiocarbónicas disponibles procedentes de la Cova de l'Avellaner. Todas ellas han sido calibradas con el software OxCal v.4.2. (BRONK RAMSEY, 2009) y la curva de calibración IntCal 13 (REIMER et alii, 2013) / Available radiocarbon dates from the Cova de l'Avellaner. All of them have been calibrated with the OxCal v.4.2 software (BRONK RAMSEY, 2009) and the IntCal 13 calibration curve (REIMER et alii, 2013).

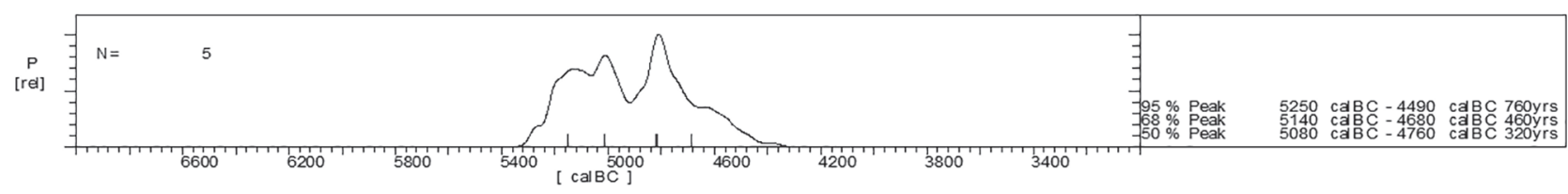

Fig. 5. Suma de las Distribuciones de Probabilidad de todas las dataciones disponibles procedentes de la Cova de l'Avellaner / Sum of Probability Distributions of all available dates from the Cova de l'Avellaner.

1 En este trabajo todos los intervalos cronológicos son presentados a $2 \sigma$. 


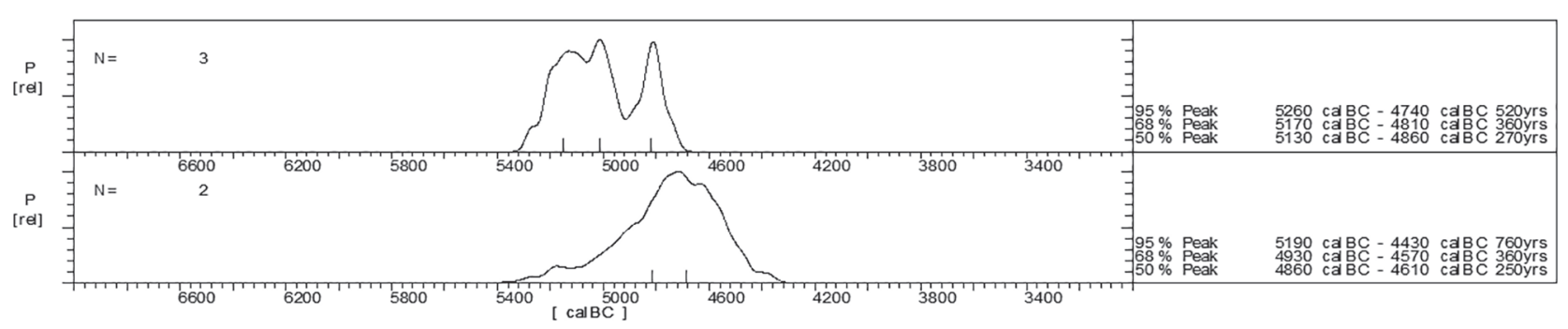

Fig. 6. Suma de las Distribuciones de Probabilidad de las dataciones radiocarbónicas realizadas en AMS (parte superior del gráfico) y por Método Convencional (parte inferior del gráfico) de la Cova de l'Avellaner / Sum of Probability Distributions of the radiocarbon dating carried out by AMS method (upper part of the graph) and by Conventional Method (lower part of the graph) of the Cova de l'Avellaner.

\section{LA COVA DE L'AVELLANER EN RELACIÓN A LOS OTROS ENTERRAMIENTOS EN CUEVA DEL NEOLÍTICO ANTIGUO Y EL NEOLÍTICO FINAL CALCOLÍTICO DEL NORESTE PENINSULAR}

Cabe decir, en primer lugar, que son muy escasos los contextos funerarios en cueva de inicios del neolítico del noreste de la península ibérica que han sido fechados. Tanto es así, que las únicas referencias publicadas que tenemos proceden de: Cova Bonica (Vallirana, Barcelona), Cova Foradada (Calafell, Barcelona), Cova del Pasteral (la Cellera del Ter, Girona) y Cova de Can Sadurní (Begues, Barcelona) (BOSCH, 1985, BLASCO et alii, 2005, OMS et alii, 2015, 2016, 2017) (Tabla 2)

Gracias a las dataciones efectuadas en la Cova de Can Sadurní o en Cova Bonica, ésta última a partir de intervenciones arqueológicas recientes, hoy tenemos constancia de la preferencia de aquellas primeras comunidades neolíticas por inhumar a sus muertos en cuevas. Hasta hace bien poco, esta cuestión era absolutamente desconocida, pues no teníamos dataciones de restos humanos para este periodo. Un gran vacío que poco a poco va llenándose como resultado del esfuerzo efectuado por ciertos equipos que han vuelto a excavar yacimientos tan complejos como Cova Bonica o han empujado baterías de dataciones en los yacimientos en los que trabajan. En todo caso, y aun siendo pocos los yacimientos con dataciones radiocarbóni- cas sobre restos humanos por AMS, nos ha parecido interesante hacer un primer test dirigido a analizar su grado de contemporaneidad teniendo en cuenta todas las fechas presentadas en la tabla 2. A este respecto, el test del Chi-Cuadrado nos ha confirmado que existe una superposición temporal entre:

- los enterramientos de la cavidad sepulcral-2 de la Cova de l'Avellaner y del nivel lc de Cova Foradada se sitúan entre el 5280-5055 cal BC (Acomb 119.5),

- los enterramientos de los niveles c10b de la Cova de Can Sadurní y el espacio III de la Cova del Pasteral entre el 4230 y el 4050 cal BC (Acomb.110.3),

- los enterramientos del nivel IV2 de Cova Bonica entre el 5465-5320 cal BC (Acomb.100.2).

Por otro lado, el análisis de la Suma de las Distribuciones de Probabilidad nos permite observar que, mientras los enterramientos de la Cova de l'Avellaner finalizarían en torno al $4700 \mathrm{cal} \mathrm{BC}$, el resto de inhumaciones en cueva del noreste peninsular no lo harían hasta el 3900 cal BC aproximadamente. Ello sugiere una discontinuidad temporal en relación a su finalización, alrededor de unos 800 años (Fig. 7). Más amplia sería todavía la discontinuidad entre las distribuciones cronológicas de este conjunto de enterramientos en cueva (5500-5000 cal BC aproximadamente) y las más recientes pertenecientes a inhumaciones del horizonte cultural del Neolítico Medio Inicial (4300-4000 cal BC).

\begin{tabular}{|l|c|c|c|c|c|c|c|c|}
\hline Yacimiento & Laboratorio & $\begin{array}{c}\text { Datación } \\
\text { BP }\end{array}$ & $\begin{array}{c}\text { Desviación } \\
\text { Estándar }\end{array}$ & Muestra & Procedencia & $\begin{array}{c}\text { Calibración } \\
\mathbf{2 \sigma}\end{array}$ & Método & $\begin{array}{c}\text { Referencia } \\
\text { Bibliográfica }\end{array}$ \\
\hline Cova Bonica & OXA29639 & 6351 & 32 & Hueso humano & IV2 & $5465-5225$ & AMS & OMS et alii, 2017 \\
\hline Cova Bonica & Beta-384726 & 6390 & 30 & Hueso humano & IV2 & $5470-5315$ & AMS & OMS et alii, 2017 \\
\hline Cova Bonica & Beta-384727 & 6410 & 30 & Hueso humano & IV2 & $5470-5325$ & AMS & OMS et alii, 2017 \\
\hline Cova Bonica & Beta-384724 & 6410 & 30 & Hueso humano & IV2 & $5470-5325$ & AMS & OMS et alii, 2017 \\
\hline Cova Foradada & Beta-248524 & 6200 & 40 & Hueso humano & IC & $5295-5045$ & AMS & OMS et alii, 2016 \\
\hline Cova Pasteral & UBAR-101 & 5270 & 70 & Hueso humano & Espacio III & $4315-3965$ & Conven-cional & BOSCH, 1985 \\
\hline Can Sadurní & Beta-197134 & 5290 & 40 & Hueso humano & c10b & $4240-3995$ & AMS & BLASCO et alii,2005 \\
\hline Can Sadurní & Beta-210652 & 5340 & 40 & Hueso humano & $10 \mathrm{~b}$ & $4320-4050$ & AMS & BLASCO et alii,2005 \\
\hline
\end{tabular}

Tabla 2: Dataciones radiocarbónicas de los enterramientos en cueva del Neolítico Antiguo del noreste peninsular. Todas ellas han sido calibradas con el software OxCal v.4.2. (BRONK RAMSEY, 2009) y la curva de calibración IntCal 13 (REIMER et alii, 2013) / Radiocarbon dates of the Early Neolithic cave burials of the north-east of the Iberian Peninsula. All of them have been calibrated with the OxCal v.4.2 software (BRONK RAMSEY, 2009) and the IntCal 13 calibration curve (REIMER et alii, 2013). 


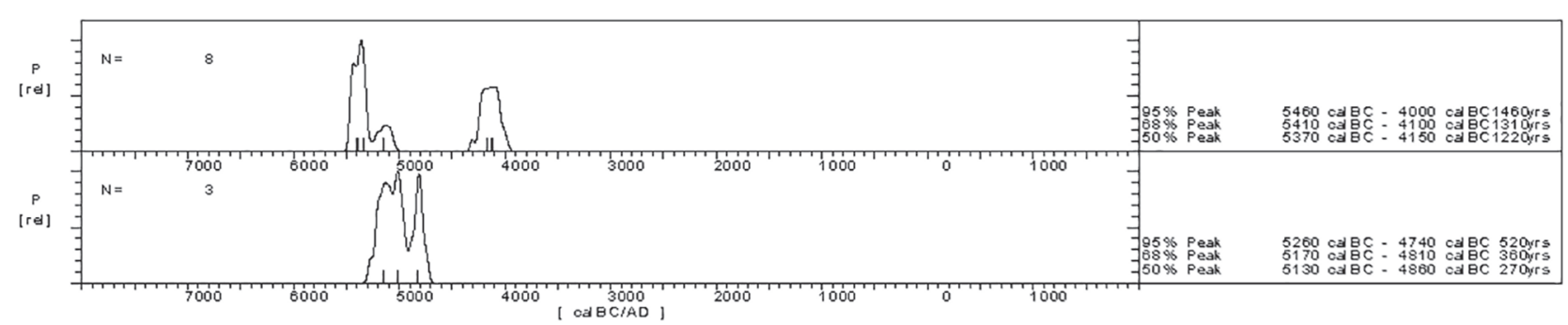

Fig. 7. Suma de las Distribuciones de Probabilidad de las dataciones procedentes de la Cueva del Avellaner (parte inferior del gráfico) y del resto de enterramientos en cueva del Neolítico Antiguo del noreste peninsular (parte superior del gráfico) / Sum of Probability Distributions of the radiocarbon of the Cueva del Avellaner (lower part of the graph) and the Neolithic cave burials of the northeast of the Iberian Peninsula (upper part of the graph).

Finalmente, nos parecía relevante examinar las dataciones existentes sobre contextos con inhumaciones en cueva a lo largo de todo el neolítico e inicios del calcolítico. Por ello, decidimos realizar nuevamente un Suma de las Distribuciones de Probabilidad comparativa de las dataciones disponibles (Fig. 8).

Los resultados obtenidos nos han permitido observar como la distribución cronológica de los enterramientos en cueva a lo largo de este periodo se sitúa, aproximadamente, entre el 5500 y el 2200 cal BC. No obstante, entre el 4000 y el 3500 cal BC se observa un vacío temporal que se corresponde, precisamente, con el horizonte de los Sepulcros de Fosa donde los enterramientos se hacen en fosas o cistas enterradas en el suelo, acogiendo mayoritariamente a un único individuo (GIBAJA et alii, 2012, OMS et alii, 2016). Aunque sólo hemos evaluado la cronología de los enterramientos efectuados en cueva, es decir teniendo como referente el contexto, es más que evidente que son enormemente relevantes las diferencias que se perciben a lo largo de todo el neolítico-calcolítico con respecto al número de inhumaciones, el tratamiento de los cuerpos, la calidad y cantidad de los ajuares y elementos personales hallados junto a los restos humanos depositados, etc. En todo caso, parece que la cueva como lugar funerario fue empleado, preferentemente, a inicios y finales del neolítico, así como durante el calcolítico.

\section{CONCLUSIONES}

El presente trabajo ha tenido un doble objetivo. Por un lado, dar a conocer las nuevas dataciones radiocarbónicas realizadas en las tres cavidades que constitu- yen la Cova de l'Avellaner y, por otro, contextualizar tales dataciones con las publicadas en otras cuevas del neolítico antiguo del noreste peninsular donde se han documentado inhumaciones.

En el caso de la Cova de l'Avellanes, los directores de la excavación (À. Bosch y J. Tarrús) sugieren que la actual horquilla cronológica a 2 sigmas (95\% de probabilidad) que nos ofrecen las cinco fechas por radiocarbono, una sobre carbones y el resto sobre huesos humanos, es demasiado amplia para los materiales arqueológicos de que disponemos. Una cronología más centrada en los valores medios de las fechas disponibles nos situaría entre 5250-4740 cal BC., un período de tiempo mucho más acorde con los datos arqueológicos de esta singular cueva sepulcral de la primera mitad del $\checkmark$ milenio cal BC. Intervalo que, a grandes rasgos, coincide con las dataciones efectuadas en las otras cuatro cuevas del neolítico antiguo de esta región donde también se han hallado inhumaciones: Cova Bonica, Cova Foradada, Cova del Pasteral y Cova de Can Sadurní.

Estos resultados nos permiten afirmar, por el momento, que el uso de las cuevas como lugar de enterramiento es una característica peculiar del neolítico antiguo. Hacia finales del $\mathrm{V}$ milenio, sin embargo, su uso funerario desaparece, momento que coincide con la implantación de otros modos de inhumación como son las tumbas individuales, y en menor medida dobles, realizadas en fosas o cistas excavadas en el suelo. Este tipo de estructuras funerarias, que son las que dan nombre al horizonte de la "Cultura de los Sepulcros de Fosa", desaparecerán igualmente hacia mediados del IV milenio, cuando aparecen otras formas y espacios de inhumación, entre las que el uso de las cue-

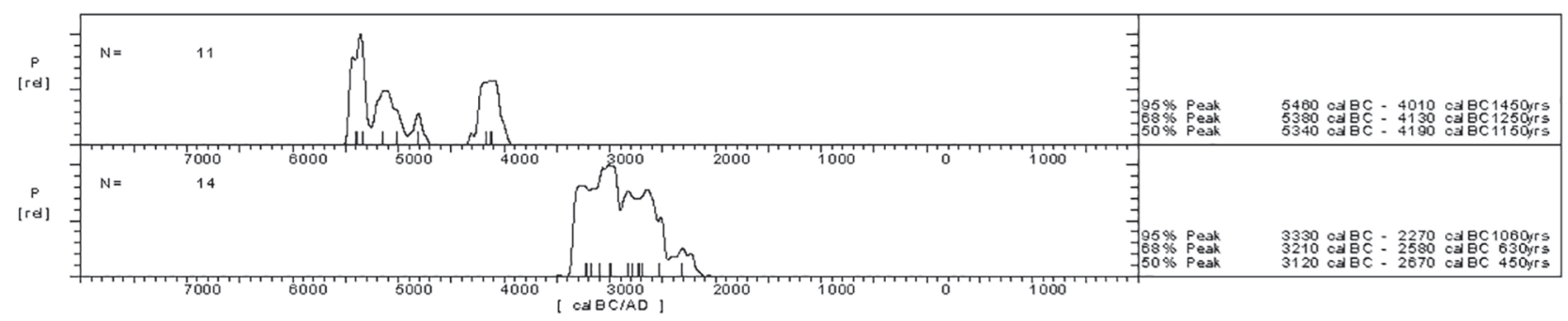

Fig. 8. Suma de las Distribuciones de Probabilidad de las dataciones procedentes de los enterramientos en cuevas del Neolítico Antiguo (parte inferior del gráfico) y Final-Calcolítico (parte superior del gráfico) del noreste de la península Ibérica / Sum of Probability Distributions of the radiocarbon dates of the Early Neolithic burials (bottom part of the graph) and the Late Neolithic-Chalcolithic ones (top part of the graph) of the northeast of the Iberian Peninsula. 
vas nuevamente vuelve a tener un papel importante. En este caso, y a diferencia de los enterramientos en fosa y cista, los enterramientos colectivos retoman su protagonismo.

Dicho todo esto, queda mucho por hacer. Será fundamental en un futuro aumentar el número de dataciones para poder tener más claro el cuadro cronológico en el que se enmarcan las distintas prácticas funerarias del neolítico en esta región.

\section{AGRADECIMIENTOS}

Este trabajo queremos dedicarlo a nuestro compañero Oriol Mercadal que recientemente nos ha dejado y que trabajó intensamente en el estudio antropológico de esta cueva. Las fechas aquí presentadas han sido financiadas por el Ministerio de Economía y Competividad, y realizadas gracias a un acuerdo de colaboración entre el CSIC, la Universitat Autònoma de Barcelona y el Centro Nacional de Aceleradores (Universidad de Sevilla-CSIC). Agradecer al Museu de la Garrotxa (Olot) y a sus conservadores su amabilidad y disponibilidad a la hora de poder tomar las muestras que iban a ser datadas. Este trabajo se ha realizado en el marco de los proyectos: Aproximación a las primeras comunidades neolíticas del NE peninsular a través de sus prácticas funerarias - HAR2011-23149-, y Aproximación a las primeras comunidades neolíticas del Mediterráneo nordoccidental: construyendo respuesta desde los análisis paleoantropológicos y genéticos - HAR2015-67323. La colaboración de Berta Morell ha sido posible gracias a una beca predoctoral (FI-DGR2014) financiada por l'Agència d'Ajuts Universitaris i de Recerca de la Generalitat de Catalunya. También desde estas líneas dar las gracias a los dos revisores anónimos que han valorado este trabajo. Sus comentarios han mejorado, sin duda, lo aquí publicado.

\section{BIBLIOGRAFIA}

Alcalde, G., Saña, M., 2009. Sis mil anys vivint a la vora dels aiguamolls de la vall d'en Bas. Amics de Besalú i el seu comtat, Besalú.

Alcalde, G., Colominas, N., Navarrete, V., Pons, E., Revelles, J., Rosillo, R., Sala, R., Saña, M., Tornero, C., Vila, O., 20132014. Vuit anys de recerca all jacimemnt de la Dou (St. Esteve d'en Bas, Garrotxa) (2006-2013): del neolític antic al bronze final. Tribuna d'Arqueologia 2013-2014, 196-209.

Ashmore, P., 1999. Radiocarbon dating: avoiding errors by avoiding mixed samples. Antiquity 73, 124-130.

Blasco, A., Edo, M., Villalba, M.J., Saña, M., 2005. Primeros datos sobre la utilización sepulcral de la Cova de Can Sadurní (Begues, Baix Llobregat) en el Neolítico Cardial. In: Arias, P., Ontañón, R., García, C. (Eds.), III Congreso del Neolítico en la Península Ibérica, 625-633, Universidad de Cantabria Servicio de Publicaciones.

Blasco, A., Edo, M., Villalba, M.J., 2011. La cova de Can Sadurní, guió sintètic de la prehistòria recent del Garraf. In: Blasco, A., Edo, M., Villalba, M.J. (coord.), La Cova de Can Sadurní i la prehistòria del Garraf, 13-95. Editorial EDAR, Arqueología y Patrimonio.

Bosch, A., 1985. La cova del Pasteral. Un jaciment neolític a la vall mitjana del Ter. Quaderns del Centre d'Estudis Comarcal de Banyoles. Homenatge al Dr. J.M. Corominas, Vol. II, 29-56.

Bosch, A., Tarrús, J., 1987. Enterramiento múltiple del Neolítico Antiguo catalán. Cova de l'Avellaner (la Garrotxa, Gerona). Revista de Arqueología 76, 20-24.

Bosch, A., Tarrús, J., 1990. La cova sepulcral del Neolític Antic de l'Avellaner (Cogolls, les Planes d'Hostoles, la Garrotxa). Centre d'Investigacions Arqueològiques de Girona, Sèrie Monogràfica $n^{\circ} 11$, Girona.

Bosch, A., Mercadal, O., Tarrús, J., 1989. La cova sepulcral del Neolític Antic de l'Avellaner (les Planes d'Hostoles, la Garrotxa). Tribuna d'Arqueologia 1988-1989, 15-27.

Bosch, À., Buxó, R., Palomo, A., Buch, M., Mateu, J., Tabernero, E., Casadevall, J., 1998. El poblat neolític de Plansallosa. L'explotació del territori dels primers agricultors-ramaders de l'Alta Garrotxa. Publicacions Eventuals d'Arqueologia de la Garrotxa, 5, Olot.

Bronk Ramsey, C., 2009. Bayesian analysis of radiocarbon dates. Radiocarbon 51(1), 337-360.

Dean, J.S., 1978. Independent dating in archaeological analysis. In: Schiffer, B. (Ed.), Advances in archaeological method and theory, 223-255. New York.

DeNiro M. J., 1985. Post-mortem preservation and alteration of in vivo bone collagen isotope ratios in relation to paleodietary reconstuction. Nature 317, 806-809.

Fontanals-Coll, M., Subirà, M.E., Diaz-Zorita, M., Gibaja, J.F., 2016. First insight into the Neolithic subsistence economy in the north-east Iberian Peninsula: paleodietary reconstruction through stable isotopes. American Journal of Physical Anthropology, DOI 10.1002/ajpa.23083

Gibaja, J.F., 2004. Prácticas funerarias durante el neolítico en Cataluña. Los enterramientos en la Península Ibérica durante la Prehistoria Reciente. Mainake 26, 9-27.

Gibaja, J.F., Subirà, M.E., Terradas, X., Fernández, E., Ruíz, J., 2012. Funerary practices during the Early-Middle Neolithic in north-east Iberia. In: Gibaja, J.F., Carvalho, A.F., Chambon, P. (Eds), Funerary practices in the Iberian Peninsula from the Mesolithic to the Chalcolithic. 29-40. British Archaeological Reports S417.

Hervella, M., Izagirre, N., Alonso, S., Fregel, R., Alonso, A., Cabrera, V.M., de la Rúa, C., 2012. Ancient DNA from Hunter-Gatherer and Farmer Groups from Northern Spain supports a Random Dispersión Model for the Neolithic Expansion into Europe. PlosOne 7, e34417. DOI: 10.1371/journalpone.033.4417.

Lacan, M., Keiser, C., Ricaud, F.X., Brucato, N., Tarrus, J., Bosch, A., Guilaine, J., Crubézy, E., Ludes, B., 2011. Ancient DNA suggest the leading role played by men in the Neolithic disseminatioon. Proceedings of de National Academy of Sciences of the United States of America 108, 18255-18259.

Molist, M., Clop, X., 2010. Los orígenes del megalitismo en Cataluña en el marco de las prácticas funerarias del Neolítico, Coloquio Internacional sobre el Megalitismo y otras manifestacions funerarias contemporáneas en su contexto social, económico y cultural. Munibe. Suplemento 32, 212-224.

Morales J.I., Fontanals M., Oms F.X., Vergès, J.M., 2010. La chronologie du Néolithique ancien cardial du nord-est de la 
Péninsule Ibérique. Datations, problématique et méthodologie. L'Anthropologie 114, 427-444.

Oms, F.X., Cebrià, A., Morales, J.I., Pedro, M., 2015. Una inhumació cardial a la cova Foradada (Calafell, Baix Penedès). In: Esteve, X., Miró, C., Molist. N., Sabaté, G. (Coords.), Jornades d'Arqueologia del Penedès, 59-64. Institut d'Estudis Penedesencs, Vilafranca del Penedès.

Oms, F.X., Martín, A., Esteve, X., Mestres, J., Morell, B., Subirà, M.E., Gibaja, J.F., 2016. The Neolithic in North-East Iberia: Chronocultural Phases and 14C. Radiocarbon 58(2), 291-309.

Oms, X., Daura, J., Sanz, M., Mendiela, S., Pedro, M., Martínez, P., 2017. First Evidence of Collective Human Inhumation from the Cardial Neolithic (Cova Bonica, Barcelona, NE Iberian Peninsula). Journal of Field Archaeology 42(1), 43-53.

Reimer, P.J., Bard, E., Bayliss, A., Beck, J.W., Blackwell, P.G., Bronk Ramsey, C., Buck, C.E., Cheng, H., Edwards, R.L., Friedrich, M., Grootes, P.M., Guilderson, T.P., Haflidason, H., Hajdas, I., Hatte, C., Heaton, T.J., Hoffmann, D.L., Hogg, A.G., Hughen, K.A., Kaiser, K.F., Kromer, B., Manning, S.W., Niu, M., Reimer, R.W., Richards, D.A., Scott, E.M., Southon, J.R., Staff, R.A., Turnery, C.S. and Van der Plich, J., 2013. IntCal13 and Marine13 radiocarbon age calibration curves $0-50,000$ years cal BP. Radiocarbon 55, 1869-1887.

Rúa, C. de la, Izagirre, N., Alonso, S., Hervella, M., 2015. Ancient DNA in the Cantabrian fringe populations: A mtDNA study from Prehistory to Late Antiquity. Quaternary International 364, 306-311.

Schiffer, M.B., 1986. Radiocarbon dating and the "old wood" problem: the case of the Hohokam chronology. Journal of Archaeological Science 13(1), 13-30.

Szécsény-Nagi, A., Roth, C., Brandt, G., Rihuete-Herrada, C., Tejedor-Rodríguez, C., Held, P., García- Martínez-de-Lagrán, I., Arcusa-Magallón, H., Zesch, S., Knipper, C., Bánffy, E., Friederich, S., Meller, H., Bueno-Ramírez, P., Barroso-Bermejo, R., de Balbín-Behrmann, R., Herrero-Corral, A.M., Flores-Fernández, R., Alonso-Fernández, C., Jiménez-Echevarria, J., Rindlisbacher, L., Oliart, C., Fregeiro, M.I., Soriano, I., Vicente, O., Micó, R., Lull, V., Soler-Díaz, J., López-Padilla, J.A., Roca de Togores-Muñoz, C., Hernández-Pérez, M.S., Jover-Maestre, F.J., Lomba-Maurandi, J., Avilés-Fernández, A., Lillios, K.T., Silva, A.M., Magalhães-Ramalho, M., Oosterbeek, L.M., Cunha, C., Waterman, A.J., Roig-Buxó, J., Martínez, A., Ponce-Martínez, J., Hunt-Ortiz, M., Mejías-García, J.C., Pecero-Espín, J.C., Cruz-Auñón-Briones, R., Tomé, T., Carmona-Ballestero, E., Cardoso, J.L., Araújo, A.C., Liesau von lettow-Vorbeck, C., Blasco-Bosqued, C., Ríos-Mendoza, P., Pujante, A., Royo-GuiIlén, J.I., Esquembre-Beviá, M.A., Dos Santos Goncalves, V.M., Parreira, R., Morán-Hernández, E., Méndez-Izquierdo, E., Vega de Miguel, J., Menduiña-García, R., Martínez-Calvo, V., López-Jiménez, O., Krause, J., Pichler, S.L., Garrido-Pena, R., Kunst, M., Risch, R., Rojo-Guerra, M.A., Haak, W., Alt, K.W., 2017. The maternal genetic make-up of the Iberian Peninsula between the Neolithic and the Early Bronze Age. Scientific Reports 7, 15644.

Tarrús, J., 2017. El poblat neolític de Ca n'lsach (Palau-saverdera, Alt Empordà.Les excavacions de 1987-1994 i de 20012003, Museu d'Arqueologia de Catalunya-Girona, Sèrie Monogràfica 27. Girona.

Weninger, B., Edinborough, K., Clare, L., Jöris, O., 2011. Concepts of probability in radiocarbon analysis. Documenta Praehistorica XXXVIII, 1-20.
Williams, A.N., 2012. The use of summed radiocabon probability distributions in archaeology: a review of methods. Journal of archaeological Science 39(3), 578-589.

Zemour, A., 2013. Gestes, espaces et temps funéraires au début du Néolithique (6e millénaire et 1ère moitié du 5e millénaire cal-BC) en Italie et en France méridionale. Reconnaissance des témoins archéologiques de l'après-mort. Thèse de I'Université de Nice Sophia Antipolis et de l'Universita di Roma-La Sapienza.

Zemour, A., Binder, D., Coppa, A., Duday, H., 2017. La sépulture au début du Néolithique (Vle millénaire et première moitié du Ve millénaire cal BC) en France méridionale et en Italie : de l'idée d'une "simple" fosse à une typologie architecturale, Bulletins et Mémoires de la société d'Anthropologie de Paris 29(1-2), 94-111.

Zilhão, J., 2001. Radiocarbon evidence for maritime pioneer colonization at the origins of farming in west Mediterranean Europe. Proceedings of the National Academy of Sciences of the United States of America 98(24), 14180-14185. 
